\title{
SER Analysis of OFDM System over Rayleigh Fading Channel
}

\author{
Mukesh Kumar Mishra \\ Department of ECE \\ National Institute of Technology Jalandhar, India
}

\author{
Neetu Sood \\ Department of ECE \\ National Institute of Technology Jalandhar, India
}

\begin{abstract}
In this paper, we present an efficient technique for the SER of OFDM system over Rayleigh fading channels, using well known characteristics function (CHF) based analysis approach. The exact probability density function of a sum of Rayleigh random vectors are used to derive the expression for the error rates of OFDM system. The average SER expressed in terms of the exponential and Bessel functions. Further to improve the system performance error-rate analysis is extended to a system using multichannel reception with maximal ratio combining.
\end{abstract}

\section{Keywords}

OFDM, Digital Communication, Rayleigh Fading channel, MRC diversity.

\section{INTRODUCTION}

ORTHOGONAL frequency-division multiplex (OFDM) is an effective technique for mitigating the effects of delay spread in frequency selective fading channels [1]. The basic principle of OFDM is to split the high data stream into number of lower rate data streams, which are transmitted simultaneously over a number of sub-carriers. Using the overlapping multi-carrier modulation technique almost $50 \%$ bandwidth can be saved [2]. In an OFDM system, IFFT and FFT processing are used in the modulation and demodulation of the signals. One important attribute of this scheme is the spreading of a fade over many bits. Rather than having a few adjacent bits completely destroyed, we now have all the bits only slightly affected by a fade [3].

Many error-rate performance studies of the OFDM systems can be found in the literature [1]-[7]. The complexity of the errorrate analysis depends on the employed digital-modulation method and the used channel-fading model. By far, the most common data-modulation method employed for the OFDM is either M-phase shift keying (PSK) or M-quadratic amplitude modulation [8]. A great number of distributions exist that well describe the statistics of the mobile radio signal [9]-[10]. Among them, the Rayleigh distribution is the basic model to characterize the fading channels.

The Bit Error Rate (BER) performance of OFDM systems communicating over Rayleigh fading channels has been extensively studied. In [11], used the pilot symbol along with the previously known channel coefficients for fast Rayleigh faded channels. Estimation of OFDM system in Rayleigh faded channel is provided by many techniques, in [12], Timing phase estimator for OFDM system in Rayleigh faded environment is proposed with low complexity. In [13] an accurate analytical solution for the bit error rate (BER) of a DS-CDMA system operating over Rayleigh fading channels has been derived. In [14] diversity at the receiver is a well-known promising avenue for improving mean signal strength and reducing signal level fluctuations in fading channels, where the multiple received copies can be combined intelligently to provide a higher average received signal-to-noise ratio (SNR).In [1]The combination of OFDM and diversity has been becoming popular in wireless communications. In [9] and [15], Proakis derives the average symbol-error probability (ASER) of MPSK with maximal-ratio combining (MRC) in Rayleigh fading. In [16] presents finiterange integral expressions for MPSK and MDPSK in Rician fading.

In this paper we present the SER analysis of QPSK-OFDM systems over Rayleigh fading channel. Our Technical contributions are summarized below. We first calculate the pdf of sum of Rayleigh random phase vectors in an integral form by using CHF method. Then we use this pdf to evaluate the error rates performance for QPSK modulated OFDM system in frequency selective Rayleigh fading channels for one tap and two-tap. We express the average SER in terms of exponential function and Bessel functions. Further to improve the system performance we use MRC diversity technique.

The rest of the paper organized as follows. The section 2 concerns with the system model. In section 3 we derive integral expression for the pdf of a sum of the random phase vectors. In section 4 SER expressions for QPSK-OFDM system over frequency selective fading channels are derived. Error-rate analysis is extended to a system using multichannel reception with maximal ratio combining in 5. The section 6 discusses about the numerical results and finally section 7 concludes the work.

\section{SYSTEM MODEL}

Consider an OFDM system with $\mathrm{N}$ sub-carriers. Let $\mathrm{X}(\mathrm{k})$ is the $\mathrm{k}^{\text {th }}$ OFDM data block to be transmitted with $\mathrm{N}$ subcarriers. These data are used to modulate $\mathrm{N}$ orthogonal sub carriers. Then IDFT is used to modulate the input signal. After modulation signal can be represented as [17]:

$$
\mathrm{x}(\mathrm{n})=\frac{1}{N} \sum_{k=0}^{N-1} X(k) \exp (j 2 \pi k n / N) \quad \mathrm{n}=0,1 \ldots . \mathrm{N}-1
$$

According to the nature of the interaction between the wave and obstacle the signal can be reflected diffracted or diffused. This phenomenon referred to as multipath propagation. The channel impulse response of a multipath fading channel is modeled as $|h(n)| e^{-j \theta(n)}$. In our analysis we assume that frequency synchronization are achieved at the receiver side $\mathrm{r}(\mathrm{n})$ can be represented in frequency domain as: 


$$
R(k)=X(k) * H(k)+N
$$

Where $N$ is an independent identically distributed (i.i.d) complex Gaussian noise component with zero mean and unit variance. The amplitude of $\mathrm{H}(\mathrm{k})$ is modeled as a Rayleigh RV with PDF.

\section{JOINT CHF \& PDF FOR RAYLEIGH RVS}

In this section, we derive an integral expression for the pdf of the amplitude of Rayleigh random phase vectors.

Consider a circularly symmetric random variable is of the form $\mathrm{Z}=\mathrm{X}_{\mathrm{n}}+\mathrm{j} \mathrm{Y}_{\mathrm{n}}$, where real and imaginary parts are zero mean independent and identically distributed (iid). The magnitude is $|z|$ and the phase $\emptyset$ is uniformly distributed over $[0,2 \pi]$.

The joint CHF of the RVs $X_{n}$ and $Y_{n}$ is defined as [19]:

$\Psi_{\mathrm{XnYn}}\left(\eta_{1}, \eta_{2}\right)=E_{\mathrm{XnYn}}\left[\exp \left(\mathrm{j} \eta_{1} \mathrm{X}+\mathrm{j} \eta_{2} \mathrm{Y}\right)\right]$

With the polar-coordinate substitution and after some simplification eq. (3) can be written as [22, eq. (27)]:

$\Psi_{\mathrm{XnYn}}\left(\eta_{1}, \eta 2\right)=\int_{0}^{\infty} f(r) \cdot\left[1 / 2 \pi \int_{0}^{2 \pi} \exp (j \eta \mathrm{r} \cos (\varnothing)) d \emptyset\right] d r$

Where $\frac{1}{2 \pi}$, the pdf of $\emptyset$. The uniform distribution of $\emptyset$ is $[0,2 \pi]$. Using the integral form of the zeroth-order Bessel function [21] i.e. $\mathrm{J}_{0}(\mathrm{z})=1 / 2 \pi \int_{0}^{2 \pi}(\exp (j \mathrm{z} \cos (\varphi) d \varphi)$.so finally equation (4) reduces to the following form:

$$
\Psi_{\mathrm{XnYn}}(\eta)=\int_{0}^{\infty} f(r) \mathrm{J}_{0}(\eta \mathrm{r}) d r
$$

This equation is used to derive the expressions for the joint $\mathrm{CHF}$ for Rayleigh distributions.

The Rayleigh joint distribution is given by

$$
f(r, \theta)=\frac{r}{\pi \Omega} \exp \left(-\frac{r^{2}}{\Omega}\right), \mathrm{r} \geq 0
$$

The $\mathrm{r}$ and $\emptyset$ independent random variables, the individual probability density functions are given by:

$$
\begin{aligned}
& f(r)=\frac{2}{\Omega} r \exp \left(-\frac{r^{2}}{\Omega}\right) \quad \mathrm{r} \geq 0 \\
& f(\emptyset)=1 / 2 \pi-\pi \leq \emptyset \leq \pi
\end{aligned}
$$

To calculate the joint CHF of $\mathrm{X}$ and $\mathrm{Y}$ we have from eq. (5):

$$
\begin{aligned}
& \Psi_{\mathrm{XnYn}}(\eta)=\int_{0}^{\infty} f(r) \mathrm{J}_{0}(\eta \mathrm{r}) d r \\
& \Psi_{\mathrm{XnYn}}(\eta)=\int_{0}^{\infty} \frac{2}{\Omega} r \exp \left(-\frac{r^{2}}{\Omega}\right) \mathrm{J}_{0}(\eta \mathrm{r}) d r
\end{aligned}
$$

Now using integral identity [20, eq. (6.631.4)] and further some simplification we have:

$$
\Psi_{\mathrm{XnY}(}(\eta)=\exp ^{-\Omega \frac{\eta^{2}}{4}} \triangleq \Psi_{\mathrm{XY}}(\eta)
$$

Where $\mathrm{J}_{0}($.$) is zeroth order Bessel function of first kind[21].$ Because the channel tap coefficients are independent, the joint CHF becomes [7]:

$$
\Psi_{\mathrm{XnYn}}(\eta)=\prod_{0}^{M-1} \exp ^{-\Omega \frac{\eta^{2}}{4}} \triangleq \Psi_{\mathrm{XY}}(\eta)
$$

The pdf of the amplitude is given by [7, eq. 11] as:

$$
f_{X Y}(r)=\mathrm{r} \int_{0}^{\infty} \Psi_{X Y}(\eta) J_{0}(\eta r) \eta d \eta
$$

$$
f_{X Y}(r)=\mathrm{r} \int_{0}^{\infty} \prod_{0}^{M-1} \exp ^{-\Omega \frac{\eta^{2}}{4}} \mathrm{~J}_{0}(\eta \mathrm{r}) \eta d \eta
$$

the integral representation of the pdf in (14) is exact, which is further used to evaluate the error rate.

\section{SER PERFORMANCE ANALYSIS USING SINGLE-CHANNEL RECEPTION}

The conditional SER of a particular modulation is given by $\mathrm{Q}$ $(\mathrm{Sr})$, where $\mathrm{Q}(\mathrm{x})=\frac{1}{\sqrt{2 \pi}} \int_{x}^{\infty} \exp \left(\frac{-t^{2}}{2}\right) d t$.Using the alternative representation of above equation as given in [25].

$$
\mathrm{Q}(\mathrm{Sr})=1 / \pi \int_{0}^{\pi / 2} \exp \left(\frac{S^{2} x^{2}}{2 \sin ^{2}(\varnothing)}\right) \mathrm{d} \emptyset
$$

The symbol error rate for QPSK is given by [23]:

$$
=\operatorname{erfc}(\sqrt{\gamma})-.25 \operatorname{erf} c^{2}(\sqrt{\gamma})
$$

Using the relationship between Q-function and erfc function [24 pp.649, eq. (F.12)], we have

$$
=2 Q(S r)-Q^{2}(S r)
$$

If the SNR is high (as is necessary for practical QPSK systems) the probability of symbol error may be approximated as:

$$
\approx 2 Q(S r)
$$

Where $\mathrm{S}$ is a factor related to symbol to noise ratio (SNR).So for our system model the average error rate, denoted by $\mathrm{P}(\mathrm{S})$, is given by:

$$
\begin{array}{r}
\mathrm{P}(\mathrm{S})=\int_{0}^{\infty} 2 \mathrm{Q}(\mathrm{Sr}) f_{X Y}(r) d r \\
\mathrm{P}(\mathrm{S})=\int_{0}^{\infty}\left[\frac{2}{\pi} \int_{0}^{\pi / 2} \exp \left(-\frac{S^{2} r^{2}}{2 \sin ^{2}(\varnothing)}\right) d \emptyset\right] f_{X Y}(r) d r \\
\mathrm{P}(\mathrm{S})=\int_{0}^{\infty}\left[\frac{2}{\pi} \int_{0}^{\frac{\pi}{2}} \exp \left(-\frac{S^{2} r^{2}}{2 \sin ^{2}(\varnothing)}\right) d \emptyset\right] \times \\
\left.\left[\mathrm{r} \int_{0}^{\infty} \Psi_{X Y}(\eta) \mathrm{J}_{0}(\eta \mathrm{r}) \eta d \eta\right)\right] d r
\end{array}
$$

To simplify the equation exchange the order of integration, so equation (21) can be rewritten as:

$$
\begin{aligned}
\mathrm{P}(\mathrm{S})=\frac{1}{\pi} \int_{0}^{\infty} \Psi_{X Y}(\eta) \eta d \eta \times \\
\quad \int_{0}^{\pi / 2}\left(\int_{0}^{\infty} r \mathrm{~J}_{0}(\eta \mathrm{r}) \exp \left(-\frac{S^{2} r^{2}}{2 \sin ^{2}(\varnothing)}\right) d r\right) d \emptyset
\end{aligned}
$$

In eq. (22) solve inner integral by using an integral identity [20, eq. (6.631.4)], so eq. (19) can be expressed as:

$$
\begin{aligned}
\int_{0}^{\pi / 2}\left(\int_{0}^{\infty} r \exp \left(-\frac{S^{2} r^{2}}{2 \sin ^{2}(\varnothing)}\right) \mathrm{J}_{0}(\eta \mathrm{r}) \mathrm{dr}\right) d \emptyset= \\
\left(\int_{0}^{\pi / 2} \frac{\sin ^{2}(\varnothing)}{S^{2}} \exp \left(-\frac{.5 \eta^{2}}{S^{2}} \sin ^{2}(\varnothing)\right) d \emptyset\right)(23) \\
=\frac{\pi}{4 S^{2}} \exp \left(-\frac{.25 \eta^{2}}{S^{2}}\right)\left(\mathrm{I}_{0}\left(\frac{.25 \eta^{2}}{S^{2}}\right)-\mathrm{I}_{1}\left(\frac{.25 \eta^{2}}{S^{2}}\right)\right.
\end{aligned}
$$

Where $I_{0}($.$) and I_{1}($.$) is modified bessels function of zeroth$ order and first order respectively[21].Substituting eq. (24) into eq. (22), we can get final SER expression for the Rayleigh fading distribution.

$$
\begin{array}{r}
\mathrm{P}(\mathrm{S})=\int_{0}^{\infty} \frac{1}{2 S^{2}} \prod_{0}^{M-1} \exp ^{-\Omega \frac{\eta^{2}}{4}} \exp \left(-\frac{.25 \eta^{2}}{S^{2}}\right) \\
\left(\mathrm{I}_{0}\left(\frac{.25 \eta^{2}}{S^{2}}\right)-\mathrm{I}_{1}\left(\frac{.25 \eta^{2}}{S^{2}}\right) d \eta\right.
\end{array}
$$


Above eq. (25) can be useful for numerical computation of SER for OFDM signals transmitted over Rayleigh channels.

\section{SER ANALYSIS OF OFDM SYSTEM WITH MRC DIVERSITY}

The conditional error rate expression for maximal ratio combining (MRC) is $Q\left(S \sqrt{\sum_{j=0}^{M-1}} r_{j}\right)$ and given by [24]

$$
Q\left(S \sqrt{ } \sum_{j=0}^{M-1} r_{j}\right)=\frac{1}{\pi} \int_{0}^{\pi / 2} \exp \left(\frac{S^{2 \sum_{j=0}^{M-1} r^{2} j}}{2 \sin ^{2}(\varnothing)}\right) d \emptyset
$$

Where $r_{j}$ is the fading amplitude in the jth branch and $S$ is a factor related to signal to noise ratio (SNR)

Averaging (26) with respect to the joint PDF of the fading amplitudes, we obtain the average error rate, denoted by $\mathrm{P}_{\mathrm{MRC}}(\mathrm{S})$, as derived in [7] for the BPSK modulated signal transmitted over Nakagami-m fading channel. Here we utilize the same expression for the QPSK signal transmitted over Rayleigh fading channel.

Using [7, eq. 39] we have error rate for QPSK-OFDM system as:

$$
\begin{gathered}
\mathrm{P}_{\mathrm{MRC}}(\mathrm{S})=\frac{1}{\pi} \int_{0}^{\pi / 2} d \emptyset\left[\prod_{0}^{M-1} \int_{0}^{\infty} \exp \left(-\frac{S^{2} r^{2} j}{2 \sin ^{2}(\varnothing)}\right) r_{j}\right] \times \\
\int_{0}^{\infty} \Psi_{X Y}^{j}(\eta) J_{0}\left(\eta r_{j}\right) \eta d \eta d r_{j} \\
\mathrm{P}_{\mathrm{MRC}}(\mathrm{S})=\frac{1}{\pi} \int_{0}^{\pi / 2} \prod_{0}^{M-1} \mathrm{M}_{j}(\varnothing) d \emptyset
\end{gathered}
$$

Where

$$
\mathrm{M}_{j}(\varnothing)=\int_{0}^{\infty} \frac{\left(\sin ^{2}(\varnothing)\right)}{S^{2}} \exp \left(-\frac{\eta \sin ^{2}(\varnothing)}{2 S^{2}}\right) \Psi_{X Y}^{j}(\eta) d \eta
$$

is the moment generating function of $r_{j}$.

\section{NUMERICAL RESULTS}

In this section the error rate performance of an OFDM system over Rayleigh fading channel is analytically evaluated. Fig.1 illustrates the average BER performance versus the SNR in a QPSK modulated OFDM system in the presence of frequency selective Rayleigh fading channel with single tap system.

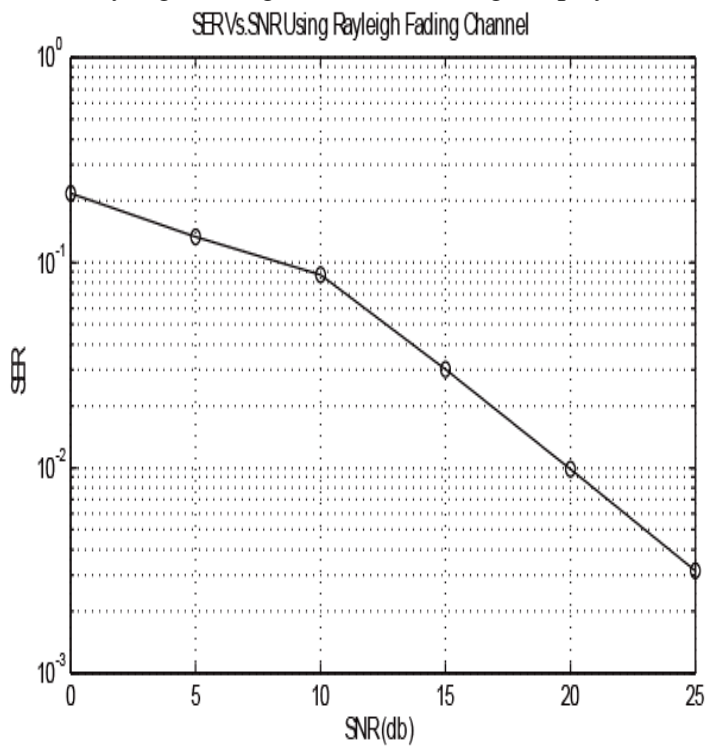

Fig.1 SER Vs SNR for OFDM system with single tap
Fig. 2 shows the error rate curves for QPSK-OFDM system over a two-tap Rayleigh fading channel with and without MRC diversity. We observe in Fig. 2 that with dual branch MRC diversity, the slopes of the error rate curves become larger and significant gains are obtained. For example, in the two-tap frequency selective Rayleigh fading channel at $\mathrm{SNR}=10$ the error rate is $15.4 \times 10^{-3}$, and with dual branch MRC reception for the same SNR we have error rate $1.44 \times 10^{-4}$.

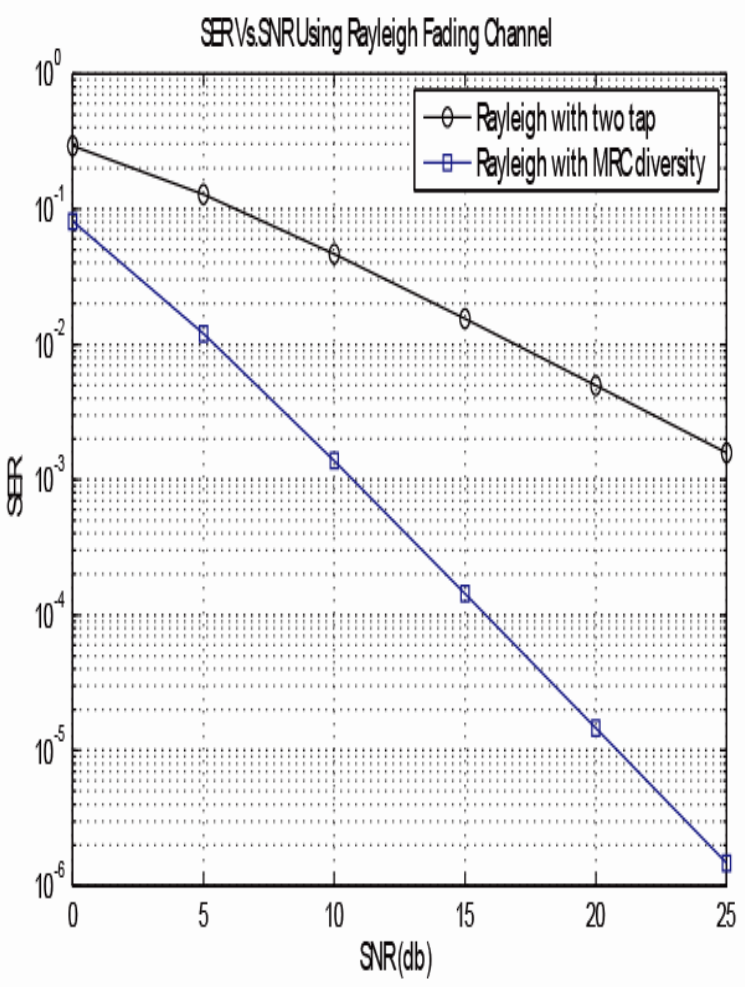

Fig.2 SER Vs SNR for OFDM system with two tap

\section{CONCLUSION}

In this paper we have investigated the error probability of OFDM systems using QPSK demodulation over frequency selective Rayleigh fading channels. Based on the joint distribution of two RVs, an explicit analytical expression for both the pdf and the SER has been derived. Basically in this paper we have outlined a simple CHF approach for deriving closed form error rates for signals in Rayleigh fading channels. Finally it has been concluded that, by using diversity technique we can improve the system performance.

\section{REFERENCES}

[1] Zhein gjiu Kang, Kung Yao, Flavio Lorenzelli, "Nakagami-m Fading Modeling in the Frequency Domain for OFDM system analysis," IEEE Communication letters, vol. 7, no.10, pp. 484-486, Oct.2003.

[2] V. Dwivedi, G. Singh "Improved BER Analysis of OFDM Communication System on Correlated Nakagami-m Fading Channel" Proceedings of International Conference on Microwave, pp. 536-539, 2008.

[3] Jun Lu, Thiang Tjhung,Fumiyuki Adachi and Cheng Li Huang, "BER performance of OFDM-MDPSK system in Frequency -Selective Rician Fading with Diversity 
Reception," IEEE Trans. On Vehicular Technology, vol. 49, no. 4, pp. 1216-1225, July 2000.

[4] T.Wang, J. G. Proakis, E. Masry, and J. R. Zeidler, "Performance degradation of OFDM systems due to Doppler spreading," IEEE Trans. Wireless Commun., vol. 5, no. 6, pp. 1422-1432, Jun. 2006.

[5] L. Wan and V. K. Dubey, "Bit error probability of OFDM system over frequency nonselective fast Rayleigh fading channels," Electron. Lett., vol. 36, no. 15, pp. 1306-1307, Jul. 2000 .

[6] Y. H. Kim, I. Song, H. G. Kim, T. Chang, and H. M. Kim, "Performance analysis of a coded OFDM system in timevarying multipath Rayleigh fading channels," IEEE Trans. Veh. Technol., vol. 48, no. 5, pp. 1610-1615, Sep. 1999.

[7] Zheng du, Julian Cheng and N.C. Beaulieu, "Accurate Error Rate Performance Analysis of OFDM on Frequency Selective Nakagami-m Fading Channels," IEEE Trans. on communications.vol. 54, no. 2, pp 319-328, Feb. 2006.

[8] Richard V. Nee, Ramjee Pmad, "OFDM for Wireless Multimedia. Communications," Artech House, 2000.

[9] John G. Proakis, "Digital Communications," McGraw-Hill, 1995.

[10] Marvin K. Simon, Mohamed- Slim Alouini," Digital Communication over Fading Channels", John Willy \& sons 2000.

[11] Jin Goog Kim,Tae Joon and Jong Tae Lim,, "Channel estimation for OFDM over Fast Rayleigh Fading Channels," Proceedings of world Academy of science and technology, vol. 21, pp. 455-458, Jan. 2007.

[12] Young Jae Ryu and Dong Seog Han, "Timing phase estimator overcoming Rayleigh Fading For OFDM systems," IEEE Proc., pp. 66-67.

[13] J. Cheng and N. Beaulieu, Accurate DS-CDMA bit-error probability calculation in Rayleigh fading, IEEE Transactions on Wireless Communications, Vol. 1, No. 1, pp. 3-15, 2002.

[14] D.A. Zogas, G. K. Karagiannidis, S. A. Kotsopoulos, "Equal Gain Combining Over Nakagami-n (Rice) and Nakagami-q (Hoyt) Generalized Fading Channels" IEEE
Trans. on Wireless Comm., vol. 4, no. 2,pp.374-379 March 2005.

[15] J. G. Proakis, "Probabilities of error for adaptive reception of M-Phase signals," IEEE Trans. Commun. Technol., vol. COM-16, pp. 71-81, Feb.1968.

[16] C. Tellambura, A. J. Mueller, and V. K. Bhargava, "Analysis of M-ary phase-shift-keying with diversity reception for land-mobile satellite channels," IEEE Trans. Veh. Technol., vol. 46, pp. 910-922, Nov. 1997.

[17] Neetu Sood, Ajay K Sharma, Moin Uddin, '’BER Performance of OFDM-BPSK and QPSK over Nakagami$m$ Fading Channels", Proc. Of $2^{\text {nd }}$ IEEE International Advance computing Conference, IACC-2010, pp. 88-90, Feb. 2010.

[18] M. Nakagami, "The m-distribution, a general formula of intensity distribution of rapid fading," in Statistical Methods in Radio Wave Propagation, W. G. Hoffman, Ed. Oxford, England: Pergamum, 1960.

[19] A. Papoulis, Probability, Random Variables, and Stochastic Processes, 3rd Ed. New York: McGraw-Hill, 1991.

[20] I. S. Gradshteyn and I. M. Ryzhik, "Table of Integrals, Series, and Products", 6th ed. San Diego, CA: Academic, 2000 .

[21] M. Abramowitz and I. A. Stegun, "Handbook of Mathematical Functions with Formulas, Graphs, and Mathematical Tables”, New York: Dover, 1972.

[22] Gregory D. Durgin, Theodore S. Rappaport, David A. de Wolf, "New Analytical Models and Probability Density Functions for Fading in Wireless Communications" ,IEEE Trans. on commu, vol. 50, no. 6, June 2002 pp.1005-1015.

[23] A. Annamalai, C. Tellambura, and V. Bhargava, "Unified analysis ofequal-gain diversity on Rician and Nakagami fading channels," in Proc. Wireless Commun. Networking Conf., 1999, pp. 10-14.

[24] Theodore S. Rappaport," Wireless Communications: Principles and Practice". Prentice-Hall, 2008.

[25] Zheng du, Julian Cheng and Norman c. Beaulieu, "Error Rate of OFDM Signals on Frequency Selective Nakagamim Fading Channels," GLOBECOM 2004 vol.06, pp. 39943998, Dec. 2004 